\title{
INFLUENCE DU MODE DE CONSERVATION DES FOURRAGES DE GRAMINEES SUR LA VITESSE DE LEUR DIGESTION DANS LE RUMEN
}

\author{
J.-P. DULPHY \\ avec la collaboration technique de Marie Jailler, J.-M. Borsseav et L. L'Hotelier \\ Station de Recherches sur l'Élevage des Ruminants, \\ Centre de Recherches de Clermont-Ferrand, I. N.R. A., \\ Saint Genès Champanelle. \\ 63110 Beaumont
}

\section{RÉSUMÉ}

I. Nous avons comparé les digestibilités en sachets (placés 13, 24, 48 et $72 \mathrm{~h}$ dans le rumen de bouvillons) d'une fétuque et d'une fléole présentes chacune sous forme d'un fourrage vert, d'un foin et de deux ensilages avec l'ingestibilité et la digestibilité de ces fourrages mesurées sur des moutons.

2. Pour une même espèce végétale les digestibilités en sachets des fourrages au bout de $4^{8}$ heures se classent comme les digestibilités mesurées in vivo.

3. Pour sept fourrages, les différences de digestibilité en sachets au bout de 13 heures rendent assez bien compte des différences d'ingestibilité surtout si on sépare les deux espèces végétales. Seule la faible ingestibilité de l'ensilage à brins longs ne peut être expliquée totalement de cette manière.

4. Cette faible ingestibilité est peut-être due à l'abaissement de la vitesse de digestion de l'ensilage à cause de la longueur des brins ou de caractéristiques physiques particulières du contenu de rumen, mais cet essai ne permet pas de conclure, et l'action d'autres facteurs peut être envisagée.

5. La capacité de digestion de la flore microbienne est plus élevée lorsque l'animal ingère des fourrages verts que lorsqu'il ingère des ensilages. La présence d'orge (avec la fléole ensilée) diminue l'activité cellulolytique de la flore du rumen.

\section{INTRODUC'TION}

La méthode des sachets de nylon (VAN KEUREN et Heinemann, I962) permet de suivre la digestion des fourrages dans le rumen. Demarquilly et Chenost (I969) ont ainsi montré que la "digestibilité 48 heures » des fourrages était étroitement liée 


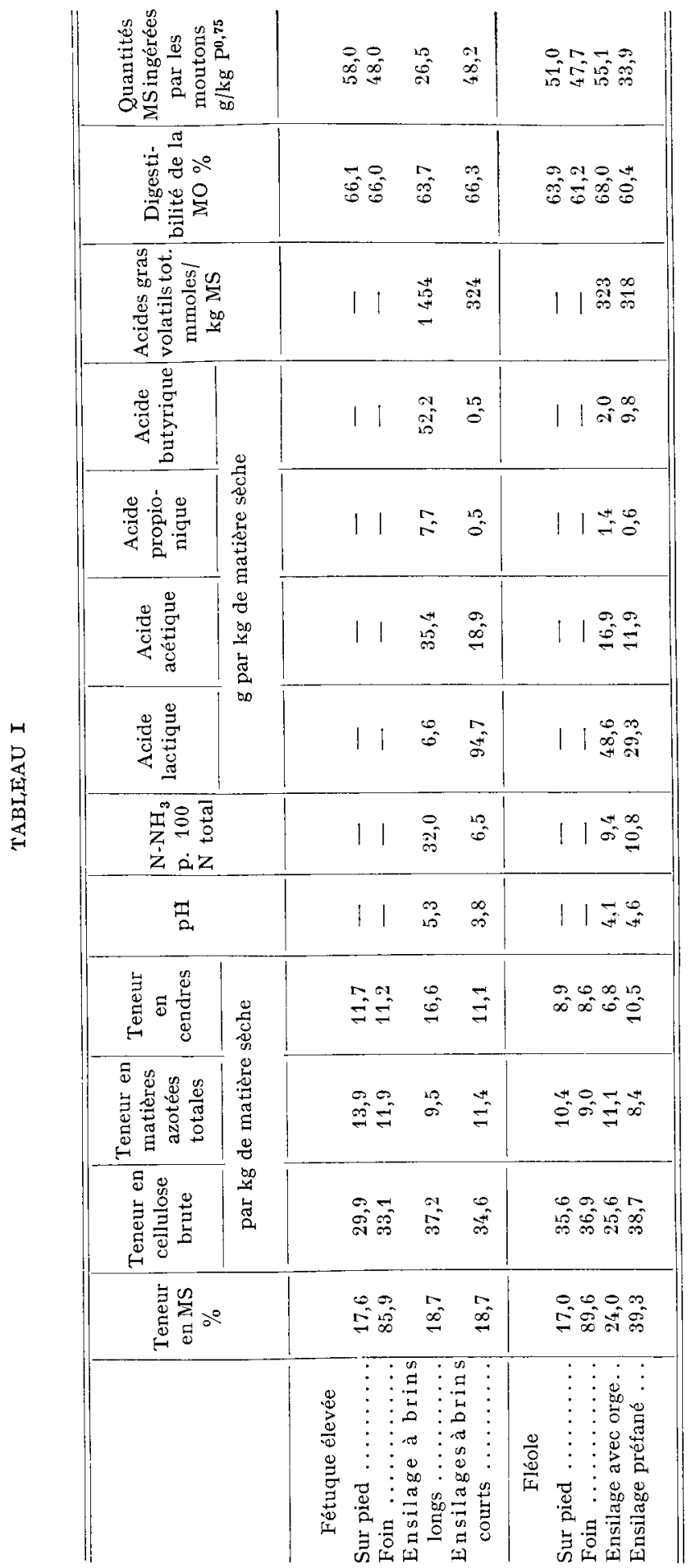


à la digestibilité in vivo et que les "digestibilités I2 et 24 heures " étaient liées aux quantités de fourrages ingérées. Il nous a paru intéressant d'examiner dans quelle mesure une diminution éventuelle de la digestibilité au cours de séjours déterminés (r2 et 48 heures) dans le rumen pouvait expliquer la diminution d'ingestibilité et de digestibilité de certains fourrages conservés par rapport aux fourrages verts initiaux. C'est pourquoi, nous avons comparé pour deux espèces végétales leurs digestibilités en sachets dans le rumen de bovins avec leurs digestibilités et leurs ingestibilités mesurées sur des moutons, cela d'une part pour les fourrages verts et d'autre part pour six fourrages conservés préparés à partir de ces derniers.

\section{MATÉRIEI, ETT MÉTHODES}

Deux fourrages verts, une fétuque élevée (variété Lutine) et une fléole (variété Mélusine), ont été récoltés en juin 1970 au stade "début épiaison ". A partir de la fétuque, on a préparé un foin ventilé et deux ensilages, l'un récolté avec une machine à fléaux (brins longs) et ensilé dircctement dans un silo en bois de $4 \mathrm{~m}^{3}$, gainé de plastique, l'autre récolté avec une machine à couteaux (brins courts) et ensilé directement dans un silo en butyl de $4 \mathrm{~m}^{3}$ également. A partir de la fléole on a préparé un foin ventilé et deux ensilages, l'un récolté avec une machine à couteaux et ensilé avec de l'orge broyée (50 p. Ioo de la matière sèche mise dans le silo) dans un silo en butyl, l'autre préfané sur le champ, haché avec une machine à couteaux et conservé dans un silo en bois gainé de plastique. Les caractéristiques des 2 fourrages verts et des 6 fourrages conservés sont données au tableau $I$.

La digestibilité de la matière organique et l'ingestibilité de ces fourrages ont été mesurées sur des moutons, selon la méthode décrite par ailleurs par DEMARquilly et JarRIgE (I964) et Demarquilly (I965).

La méthode d'étude cinétique de la digestion dans le rumen a été décrite par DEMARQUILLY et Chenost (1969). Une petite quantité (environ $3 \mathrm{~g}$ de matière sèrhe) du fourrage étudié, séché pendant 24 heures à l'étuve $\left(80^{\circ} \mathrm{C}\right)$ et broyé à la grille de $0,8 \mathrm{~mm}$, est placée dans des sachets de nylon qui sont introduits dans le rumen après le repas et retirés au bout de $\mathbf{2}$ heures (en fait I $3 \mathrm{~h}$ dans la présente expérience), 24,48 et 72 heures pour suivre la disparition de la matière sèche. Le plan d'étude est rapporté au tableau 2 ; une paille d'avoine a servi de référence dans toutes les séries de mesure pour estimer les variations de l'activité cellulolytique du jus de rumen. Les animaux utilisés étaient des bouvillons de $250 \mathrm{~kg}$ porteurs d'une fistule du rumen et recevant chaque régime depuis ro jours au moins. Deux animaux ont été utilisés durant l'été ; l'un a dû être remplacé pour les études hivernales.

La digestibilité du fourrage en sachets ainsi mesurée au bout d'un temps déterminé est la résultante de deux facteurs : l'aptitude propre du fourrage à être digéré et la capacité de digestion de la flore microbienne.

L'aptitude du fourrage à être digéré dépend d'abord de la proportion de la partie soluble (matière sèche soluble dans l'eau), puis de la vitesse de digestion de la partie insoluble (matière sèche insoluble dans l'eau). Cette aptitude a été caractérisée pour chaque temps de séjour par la moyenne des digestibilités en sachets de ce fourrage mesurées lorsque les animaux recevaient les 3 régimes correspondant à une même espèce fourragère (foin et 2 ensilages).

Nous avons caractérisé la capacité de digestion de la population microbienne correspondant à chaque régime soit par la digestibilité en sachets du fourrage témoin (paille), soit par la moyenne des digestibilités en sachets des cinq substrats (paille d'avoine, fourrage vert, foin et les deux ensilages correspondants) placés en même temps dans le rumen des animaux recevant ce régime.

Enfin, nous appellerons digestibilité « réelle " du fourrage sa digestibilité, mesurée en sachets, lorsque l'animal consomme ce même fourrage.

L'extrait aqueux des différents substrats étudiés a été mesuré sur une prise de $4 \mathrm{~g}$ de matière sèche du fourrage broyé qui a subi deux extractions par ıoo $\mathrm{ml}$ d'eau à $40^{\circ} \mathrm{C}$, la première d'une heure et la deuxième de 30 minutes.

Notons enfin, que toutes les matières sèches ont été mesurées après séchage à l'étuve $\left(t=30^{\circ} \mathrm{C}\right)$ et que les chiffres obtenus n'ont pas été corrigés pour les pertes en matières volatiles. 
TABLEAU 2

Plan d'étude des digestibilités en sachets

\begin{tabular}{|c|c|c|c|c|c|c|c|c|}
\hline \multirow{3}{*}{$\begin{array}{l}\text { Substrats mis } \\
\text { dans les sachets }\end{array}$} & \multicolumn{8}{|c|}{ Régime des animaux } \\
\hline & \multicolumn{4}{|c|}{ Fétuque } & \multicolumn{4}{|c|}{ Fléole } \\
\hline & $\begin{array}{c}\text { Fourrage } \\
\text { vert }\end{array}$ & Foin & $\begin{array}{c}\text { Ensilage } \\
\text { à brins } \\
\text { longs }\end{array}$ & $\begin{array}{l}\text { Ensilage } \\
\text { à brins } \\
\text { courts }\end{array}$ & $\begin{array}{c}\text { Fourrage } \\
\text { vert }\end{array}$ & Foin & $\begin{array}{l}\text { Ensilage } \\
\text { avec orge }\end{array}$ & $\begin{array}{l}\text { Ensilage } \\
\text { préfané }\end{array}$ \\
\hline Paille & $\mathrm{x}$ & $\mathrm{x}$ & $\mathrm{X}$ & $\mathrm{x}$ & $\mathrm{x}$ & $\mathrm{x}$ & $\mathrm{x}$ & $\mathrm{x}$ \\
\hline $\begin{array}{l}\text { Fétuque } \\
\text { Fourrage vert } \ldots \ldots \ldots \ldots \ldots \\
\text { Foin } \ldots \ldots \ldots \ldots \ldots \ldots \ldots \\
\text { Ensilage à brins longs } \ldots \ldots \\
\text { Ensilage à brins courts } \ldots\end{array}$ & $\mathrm{x}$ & $\begin{array}{l}X \\
X \\
X \\
X\end{array}$ & $\begin{array}{l}\mathrm{X} \\
\mathrm{X} \\
\mathrm{X} \\
\mathrm{X}\end{array}$ & $\begin{array}{l}X \\
X \\
X \\
x\end{array}$ & & & & \\
\hline $\begin{array}{l}\text { Fléole } \\
\text { Fourrage vert } \ldots \ldots \ldots \ldots \\
\text { Foin } \ldots \ldots \ldots \ldots \ldots \ldots \\
\text { Ensilage avec orge } \ldots \ldots \ldots \\
\text { Ensilage préfané } \ldots \ldots \ldots\end{array}$ & & & & & $\mathrm{x}$ & $\begin{array}{l}\mathrm{X} \\
\mathrm{X} \\
\mathrm{X} \\
\mathrm{X}\end{array}$ & $\begin{array}{l}\mathrm{X} \\
\mathrm{X} \\
\mathrm{X} \\
\mathrm{X}\end{array}$ & $\begin{array}{l}\mathrm{X} \\
\mathrm{X} \\
\mathrm{X} \\
\mathrm{X}\end{array}$ \\
\hline
\end{tabular}

TABLEAU 3

Fétuque : digestibilité de la matière sèche en sachets

- Digestibilité in vivo de la MO

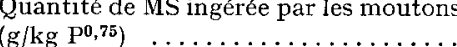

- Extrait aqueux ...................

Capacité de la digestion de la flore microbienne :

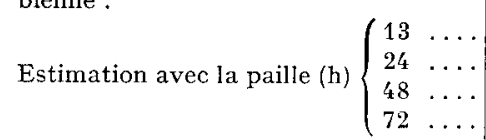

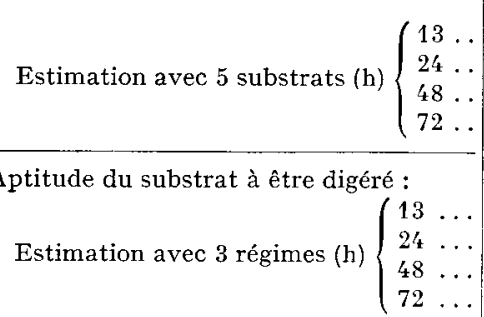

Digestibilité " réelle " $\left(\begin{array}{l}13 \ldots \ldots \ldots \ldots \\ 24 \ldots \ldots \ldots \ldots\end{array}\right) \begin{array}{r}54,4 \\ 23,3\end{array}$ du substrat $(\mathrm{h})$ :

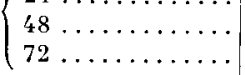

\begin{tabular}{|c|c|c|c|}
\hline $\begin{array}{c}\text { Fourrage } \\
\text { vert }\end{array}$ & Foin & $\begin{array}{l}\text { Ensilage } \\
\text { à brins longs }\end{array}$ & $\begin{array}{l}\text { Ensilage } \\
\text { à brins courts }\end{array}$ \\
\hline 66,1 & 66,0 & 63,7 & 66,3 \\
\hline 58,0 & 48,0 & 26,5 & 48,2 \\
\hline 27,6 & 23,3 & 15,7 & 22,9 \\
\hline 33,7 & 29,6 & 28,8 & 27,5 \\
\hline 48,1 & 40,0 & 37,4 & 39,2 \\
\hline 69,4 & 60,4 & 60,4 & 61,1 \\
\hline 73,9 & 70,2 & 66,8 & 68,3 \\
\hline- & 43,2 & 45,2 & 42,8 \\
\hline 一 & 56,9 & 55,9 & 54,8 \\
\hline 一 & 72,0 & 70,8 & 70,6 \\
\hline- & 77,3 & 75,0 & 75,8 \\
\hline 54,0 & 48,6 & 40,7 & 46,8 \\
\hline 67,2 & 60,4 & 53,4 & 59,4 \\
\hline 77,7 & 73,6 & 68,9 & 74,9 \\
\hline 81,0 & 77,6 & 73,8 & 79,4 \\
\hline 54,4 & 47,0 & 41,8 & 45,3 \\
\hline 73,3 & 62,5 & 51,9 & 56,7 \\
\hline 82,3 & 74,0 & 68,8 & 73,9 \\
\hline 85,0 & 77,7 & 72,3 & 78,6 \\
\hline
\end{tabular}




\section{RÉSULTATS}

\section{Fétuque (tabl. 3)}

Les digestibilités de la matière organique mesurées sur des moutons sont identiques pour trois des fourrages; seul l'ensilage à brins longs est moins digestible. Les quantités ingérées sont plus variables : par rapport au fourrage vert les diminunutions sont de $I 7,5 \mathrm{p}$. Ioo pour le foin et l'ensilage à brins courts et de $54 \mathrm{p}$. Ioo pour l'ensilage à brins longs.
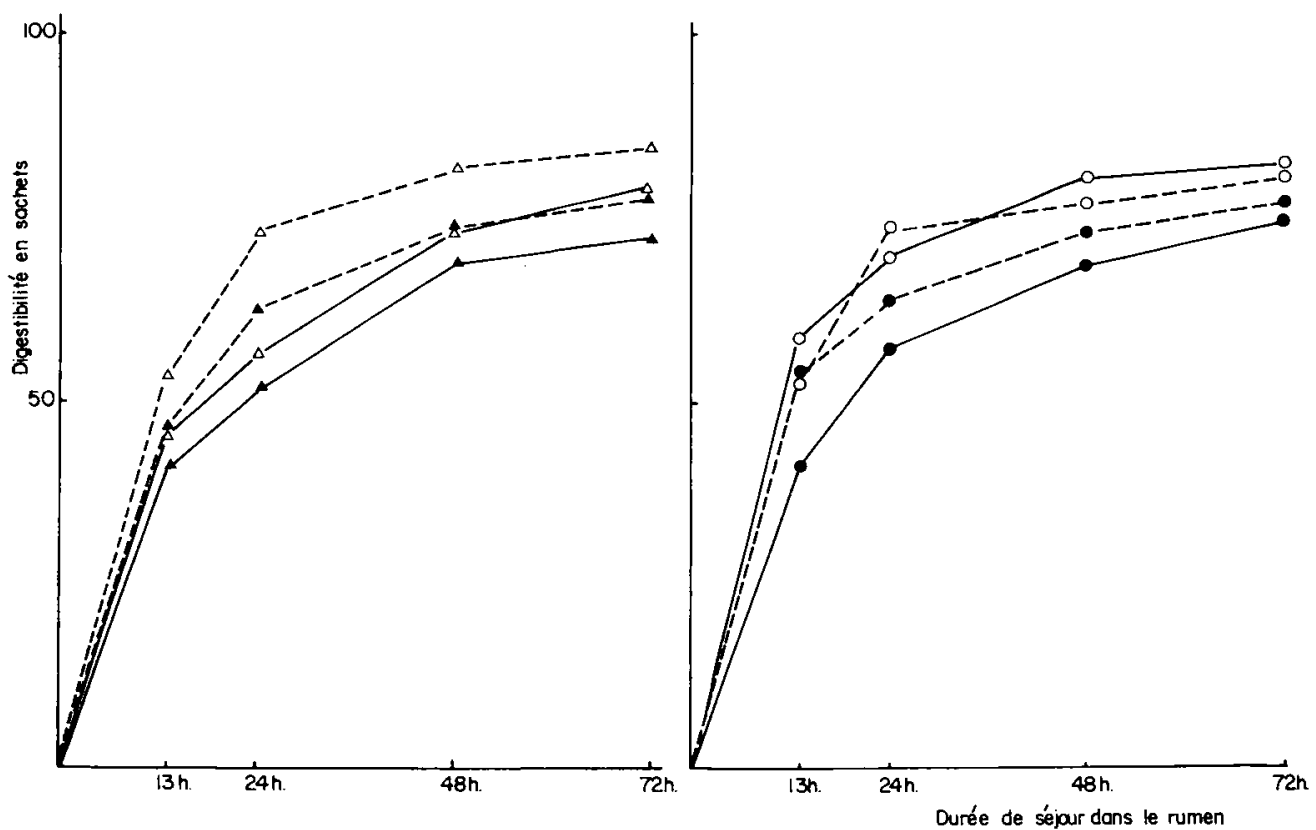

FIG. I. - Cinétique des digestibilités véelles en sachets

\section{Fétuque}

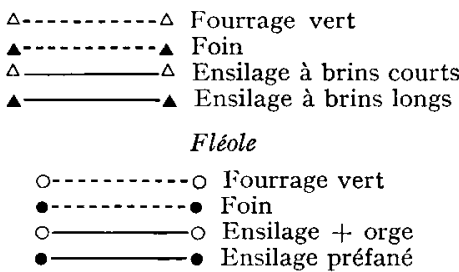

L'aptitude des fourrages à être digérés est différente surtout au temps " 13 heures " et varie dans le même sens que la digestibilité sur moutons; elle est la plus élevée pour le fourrage vert, la plus faible pour l'ensilage à brins longs et intermédiaire pour le foin et l'ensilage à brins courts. Les vitesses de digestion caracté- 
risées par le rapport $\frac{\text { Digestibilité I3 heures }}{\text { Digestibilité } 48 \text { heures }}$ sont également différentes : 69,5 p. Ioo pour le fourrage vert, $66,0 \mathrm{p}$. Ioo pour le foin, $62,5 \mathrm{p}$. Ioo pour l'ensilage à brins courts et $59 \mathrm{p}$. Ioo pour l'ensilage à brins longs. Notons que les variations de l'aptitude des fourrages à être digérés sont parallèles à celles de leur teneur en extrait aqueux.

Estimée d'après la digestibilité en sachets de la paille, la capacité de digestion de la flore microbienne est plus élevée avec le régime de fourrage vert mais elle n'est pas différente entre les trois fourrages conservés. Il en est de même pour ces trois fourrages si on prend comme critère l'estimation avec les 5 substrats.

Les digestibilités " réelles " des différents fourrages se classent de la même façon que leur aptitude à être digérés. La valeur plus élevée pour le fourrage vert est accentuée par la plus grande capacité de digestion de la flore microbienne (fig. I).

\section{Fléole (tab1. 4)}

Les digestibilités de la matière organique des 4 fourrages sont assez différentes et sont comprises entre 60,4 pour l'ensilage préfané et 68,0 pour l'ensilage contenant de l'orge. Les quantités ingérées sont également variables : par rapport au fourrage

TABLEAU 4

Fléole: digestibilité de la matière sèche en sachets

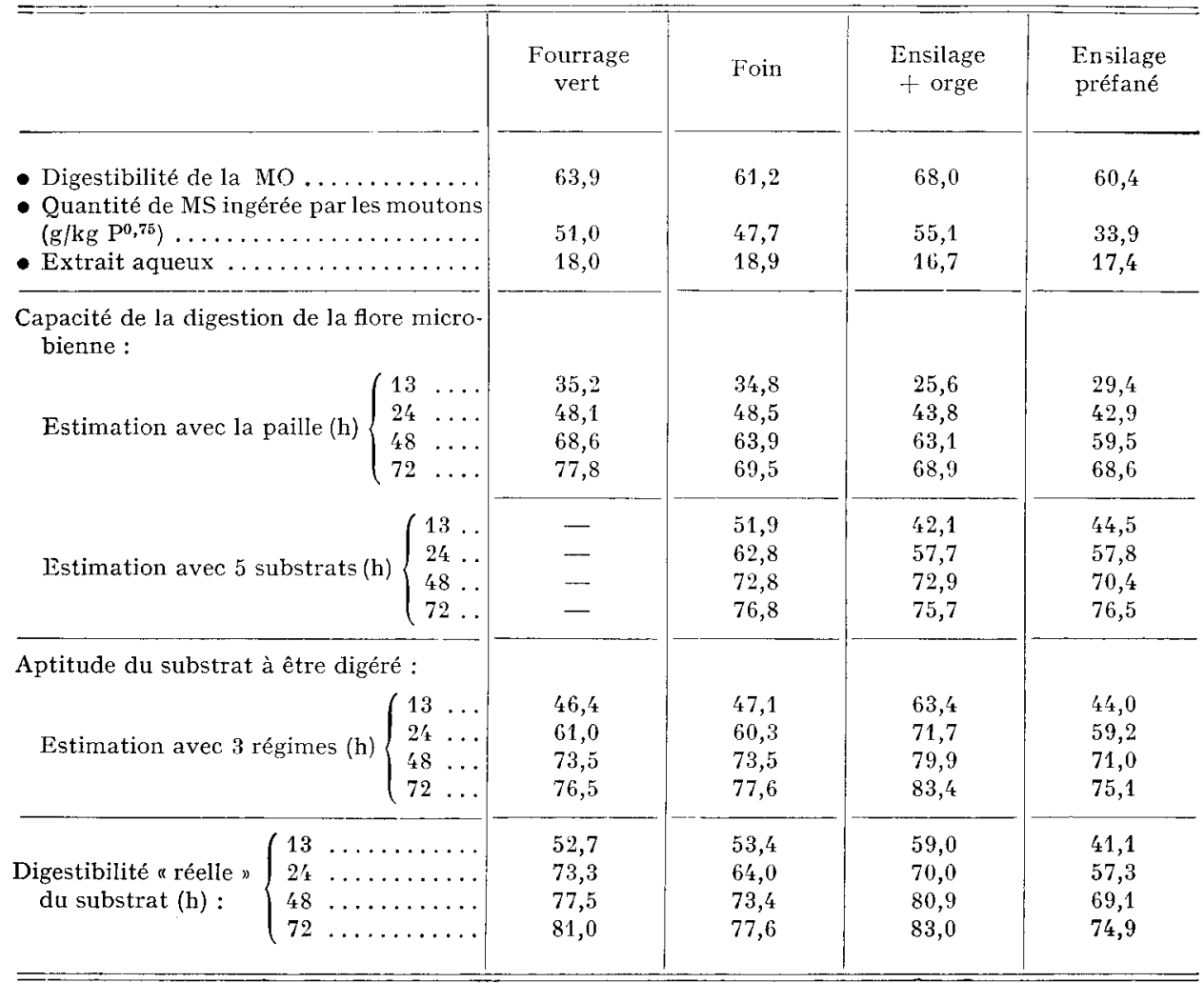


vert elles sont de $94 \mathrm{p}$. Ioo pour le foin, I08 p. Ioo pour l'ensilage contenant de l'orge et $66,5 \mathrm{p}$. Ioo pour l'ensilage préfané.

L'aptitude des 4 fourrages à être digérés varie comme la digestibilité in vivo. La vitesse de digestion mesurée par le rapport $\frac{\text { Digestibilité } \mathbf{I} 3 \text { heures }}{\text { Digestibilité } 48 \text { heures }}$ est du même ordre de grandeur pour le fourrage vert, le foin et l'ensilage préfané (respectivement 63 p. IOO, 64 p. Ioo et $62 \mathrm{p}$. IOO) ; elle est plus élevée pour l'ensilage avec orge $(79,5$ p. IOO) qui se distingue donc nettement des trois autres fourrages.

Esstimée d'après la digestibilité en sachets de la paille, la capacité de digestion de la flore microbienne est supérieure avec le régime de fourrage vert et inférieure avec le régime d'ensilage préfané. Le foin et l'ensilage contenant de l'orge conduisent à des résultats intermédiaires ; il est intéressant de noter le démarrage plus lent de l'attaque de la paille quand il y a de l'orge dans la ration. Les conclusions sont les mêmes si on considère les estimations faites à partir de 5 substrats.

Les digestibilités " réelles " au bout de I3 heures sont identiques pour le foin et le fourrage vert. Celle de l'ensilage avec de l'orge est supérieure et celle de l'ensilage préfané est inférieure. Au bout de 48 heures les digestibilités " réelles " en sachets sont différentes, celle du foin devenant inférieure à celle du fourrage vert (fig. I).
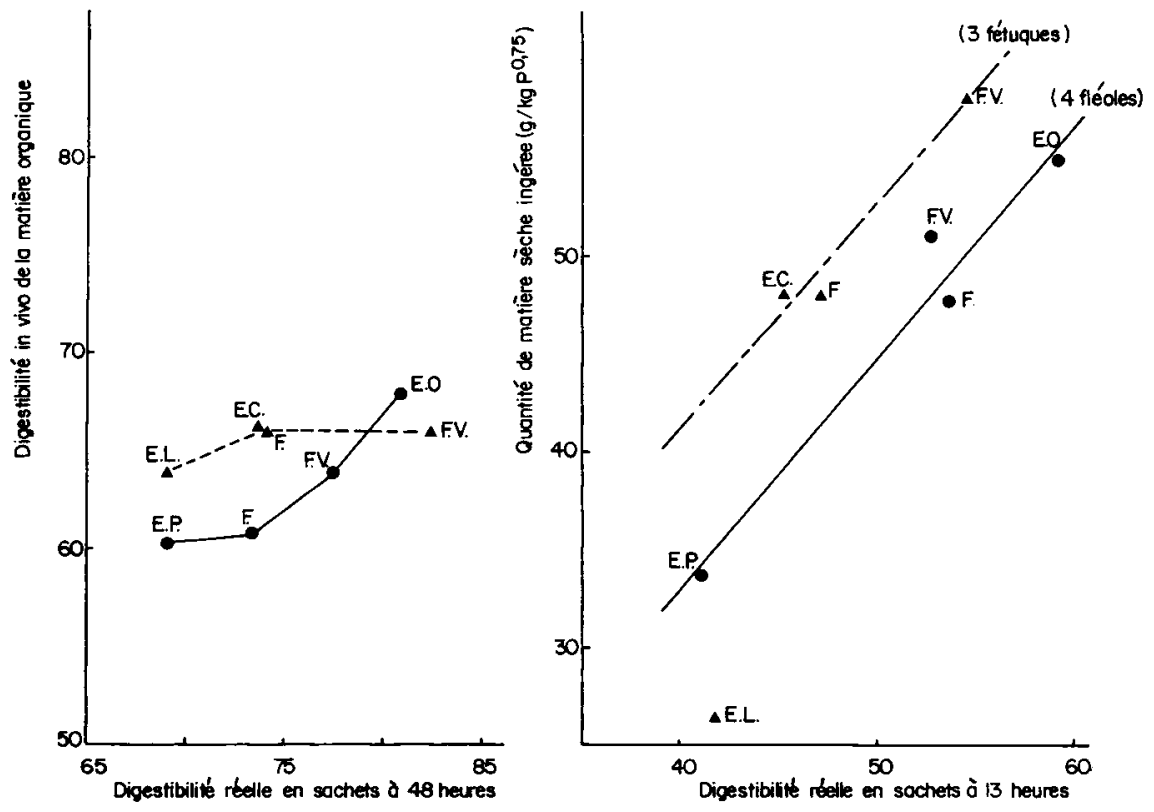

FIG. 2. - Relations entre la valeur alimentaire et la digestibilité en sachets

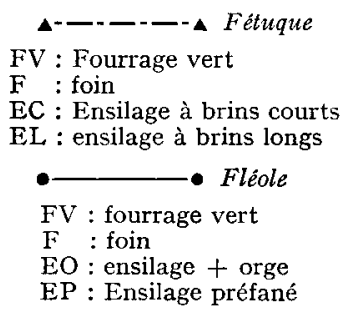




\section{Relations entre les digestibilités, les quantités ingérées mesurées sur moutons \\ et les digestibilités en sachets (fig. 2)}

Dans notre essai, les digestibilités de la matière organique des fourrages mesurées sur moutons se classent dans le même ordre que les digestibilités " réelles " en sachets au bout de 48 heures. Pour les huit fourrages la liaison est cependant peu nette $(r=+0,67 ; \mathrm{P}<0,075)$.

Les quantités ingérées sont liées aux digestibilités " réelles " en sachets au bout de 13 heures $\left(r=0,84\right.$; pente de la droite de régression, $b=\mathrm{I}, 38 \mathrm{~g} / \mathrm{kg} \mathrm{P}^{0,75}$ pour I point de digestibilité " réelle ", $\mathrm{P}<\mathrm{O}, \mathrm{OI}$ ). Pour les quatre fourrages de fléole la liaison est étroite $(r=+0,98, b=\mathrm{I}, 20, \mathrm{P}<0,02)$; ce n'est pas le cas pout les fourrages de fétuque $(r=0,88 ; \mathrm{P}<0, \mathrm{I} 2)$. Pour ces fourrages de fétuque, la pente de la droite de régression est très élevée $(b=2,2 \mathrm{I})$; par contre, si on retire l'ensilage à brins longs, la pente de la droite de régression est proche de celle relative aux fourrages de fléole ( $b=\mathrm{I}, \mathrm{I} 6)$. Il semble donc que l'ensilage de fétuque à brins longs ait été ingéré en quantité plus faible que celle qui était prévisible à partir de sa digestibilité en sachets à I3 heures.

\section{DISCUSSION}

Les différences de la digestibilité " réelle " en sachets des fourrages au bout de 48 heures rendent donc, en partie, compte des modifications de la digestibilité sur moutons entraînées par les différents mode de conservation, mais elles sont beaucoup plus importantes.

La baisse d'ingestibilité entraînée par la conservation s'explique assez bien pour les 2 foins et 3 des 4 ensilages par les diminutions de la digestibilité " réelle " à I3 heures en sachets ou par celles de la vitesse de digestion des fourrages : les variations des digestibilités "I 3 heures " sont importantes alors que celles des digestibilités " 48 heures ", de même que celles des digestibilités in vivo, sont plus faibles. Seule la faible ingestibilité de l'ensilage à brins longs quand on la compare à celle du fourrage vert et des 2 fourrages conservés correspondants ne peut être expliquée totalement par la faible digestibilité à I 3 heures en sachets, c'est-à-dire par la diminution de sa vitesse de digestion dans le rumen par rapport aux autres fourrages, diminution liée vraisemblablement à la faible quantité d'extrait aqueux de ce fourrage.

D'autres facteurs sont donc intervenus pour accentuer la faible ingestibilité de cet ensilage à brins longs dont la teneur en acides gras volatils est très élevée : soit des facteurs buccaux (goût, odeur, préhensibilité...), soit des facteurs métaboliques, ou bien des facteurs digestifs non mesurés par la technique des sachets de nylon telle que nous l'avons employée. En effet, la digestibilité en sachets est mesurée sur des fourrages broyés et mis dans des sachets lestés localisés au fond du rumen. Or, CAmpliNG ( 1965 ) note que le contenu du rumen des animaux ingérant des ensilages à brins longs est différent de celui des animaux recevant d'autres fourrages et notamment des foins; il se présente comme un enchevêtrement de fibres 
flottant à la surface de la phase liquide du rumen. L,es fibres ne baignant pas dans le jus, leur digestion est peut-être lente et de ce fait la quantité d'ensilage ingérée plus faible que celle théoriquement permise par la vitesse de digestion "intrinsèque " de l'ensilage. D'après la digestibilité à I3 heures en sachets, l'ingestibilité de cet ensilage aurait dû être d'environ $42,5 \mathrm{~g} / \mathrm{kg} \mathrm{P}^{0,75}$. C'est probablement ce qu'on aurait obtenu s'il avait été haché juste avant l'ingestion. En effet, le hachage avant la distribution aux animaux de 2 autres ensilages de fétuque récoltés au même stade et avec la même machine de récolte, a permis de faire passer leur ingestibilité de $26,3 \mathrm{~g}$ (identique à celle de l'ensilage considéré dans cette étude) à $40,5 \mathrm{~g} / \mathrm{kg} \mathrm{P}^{0,75}$.

Il est donc possible que la vitesse de digestion dans le rumen des ensilages à brins longs, et par conséquent leurs quantités ingérées, soient limitées par les caractéristiques physiques particulières du fourrage. Cet essai ne permet pas de conclure; il semble simplement que la vitesse de digestion fixe un certain niveau d'ingestion possible et que d'autres facteurs interviennent pour limiter les quantités ingérées au-dessous de ce niveau.

Enfin, il ressort de nos résultats un fait intéressant en ce qui concerne la capacité de digestion de la flore microbienne. Cette capacité de digestion dépend en partie de la nature et de la digestibilité du fourrage ingéré par l'animal ; pour des fourrages de même origine elle est plus élevée quand l'animal ingère des fourrages verts, et dans une moindre mesure des foins, que lorsqu'il ingère des ensilages. Les différences entre régimes sont par ailleurs d'autant plus importantes que la capacité de digestion est jugée par la digestion après des temps de séjour plus courts dans le rumen. Elles doivent donc jouer davantage sur l'ingestibilité des fourrages que sur leur digestibilité in vivo. Malgré la digestibilité élevée de l'ensilage contenant 50 p. roo d'orge, la capacité de digestion de la flore ruminale des animaux recevant cet ensilage est faible vis-à-vis de celui-ci au début de son séjour dans le rumen. Plus précisément, c'est l'activité cellulolytique de la flore qui est faible, car la digestibilité en sachets de l'ensilage contenant l'orge est élevée. Cette faible activité cellulolytique du jus de rumen des animaux recevant une ration riche en aliments concentrés doit expliquer, au moins en partie, les diminutions constatées par de nombreux auteurs, de l'ingestibilité et de la digestibilité des fourrages au fur et à mesure que la proportion d'aliment concentré dans la matière sèche totale de la ration augmente.

Rę̧u pour publication en avril 1972.

SÜMMARY

INFLUENCE OF THE CONSERVATION METHOD OF GREEN FORAGES

ON THEIR DIGESTION RATE IN THE RUMEN

I. The digestibilities in nylon bags (placed $\mathrm{I} 3,24,48$ and $72 \mathrm{~h}$ in the rumen of yearling steers) of one variety of fescue and one of timothy grass, each in the form of either green forage, hay or of two silages, were compared with the voluntary intake and digestibilities of these forages measured on mature castrated sheep.

2. For the same variety, the $4^{8} \mathrm{~h}$-digestibilities of the forages in nylon bags are closely related to those measured in vivo using sheep. 
3. For seven forages, the differences as regards the $13 \mathrm{~h}$-digestibilities in nylon bags demonstrate rather well the differences in the volontary intake, particulary if the two varieties are separated. Only the low voluntary intake of the "long " silage cannot be entirely explained in this way.

4. This low voluntary intake may depend on the lower rate of digestion of "long "silage due to the length of the material or to particular physical characteristics of the rumen content, but no conclusion can be drawn from this trial and the action of other factors could be involved.

5. The digestive ability of the bacterial flora is higher when the animals are fed green forages than when they receive silages. The presence of barley (with the ensiled timothy grass) reduces the cellulolytic activity of the rumen flora.

\section{RÉFÉRENCES BIBLIOGRAPHIQUES}

Campling R. C., Murdoch J. C., 1965. The effect of length of silage on its voluntary intake by cattle. J. Brit. Grassland Soc. 20, 54-58.

Demarquilly C., I965. Factors affecting the voluntary intake of green forage by sheep. Proc. 9th Inter. Grassland Congress. Sao Paulo, Section 9, p. 400 ; pp. 877-885.

Demarouilix C., Chenost M., I969. Étude de la digestion des fourrages dans le rumen par la méthode des sachets de nylon. Liaisons avec la valeur alimentaire. Ann. Zootech., 18, 4I9-436.

DEMARQUilly C., JARRIGE R., 1964. Valeur alimentaire de l'herbe des prairies temporaires aux stades d'exploitation pour le pâturage. I. Composition chimique et digestibilité. Ann. Zootech., 13, $301-339$.

Dulphy J. P., Demarquilly $\mathrm{C}$., I972. Influence de la machine de récolte sur la valeur alimentaire des ensilages. I. Resultats preliminaires Ann. Zootech. 21 I63-173.

VAn Keuren R. W., Heinemann W. W., I962. Study of a nylon bag technique for in vivo estimation of forage digestibility. J. anim. Sci., 21, 340-345. 